\title{
Applying Graph Theory and Mathematical-Computational Modelling to Study a Neurophysiological Circuit
}

\author{
Camila de Andrade Kalil1¹, Maria Clícia Stelling de Castro',2, Dilson Silva ${ }^{1,3}$, Célia Martins Cortez ${ }^{1,3}$ \\ ${ }^{1}$ Postgraduate Program in Computational Sciences, Rio de Janeiro State University, Rio de Janeiro, Brazil \\ ${ }^{2}$ Department of Informatics and Computer Science, Rio de Janeiro State University, Rio de Janeiro, Brazil \\ ${ }^{3}$ Department of Applied Mathematics, Rio de Janeiro State University, Rio de Janeiro, Brazil \\ Email: ccortezs@ime.uerj.br
}

How to cite this paper: de Andrade Kalil, C., de Castro, M.C.S., Silva, D. and Cortez, C.M. (2021) Applying Graph Theory and Mathematical-Computational Modelling to Study a Neurophysiological Circuit. Open Journal of Modelling and Simulation, 9, 159-171.

https://doi.org/10.4236/ojmsi.2021.92011

Received: February 10, 2021

Accepted: April 26, 2021

Published: April 29, 2021

Copyright (c) 2021 by author(s) and Scientific Research Publishing Inc. This work is licensed under the Creative Commons Attribution International License (CC BY 4.0).

http://creativecommons.org/licenses/by/4.0/

\section{(c) (i) Open Access}

\begin{abstract}
The aim of the present study is to contribute to the knowledge about the functioning of the neuronal circuits. We built a mathematical-computational model using graph theory for a complex neurophysiological circuit consisting of a reverberating neuronal circuit and a parallel neuronal circuit, which could be coupled. Implementing our model in $\mathrm{C}++$ and applying neurophysiological values found in the literature, we studied the discharge pattern of the reverberant circuit and the parallel circuit separately for the same input signal pattern, examining the influence of the refractory period and the synaptic delay on the respective output signal patterns. Then, the same study was performed for the complete circuit, in which the two circuits were coupled, and the parallel circuit could then influence the functioning of the reverberant. The results showed that the refractory period played an important role in forming the pattern of the output spectrum of a reverberating circuit. The inhibitory action of the parallel circuit was able to regulate the reverberation frequency, suggesting that parallel circuits may be involved in the control of reverberation circuits related to motive activities underlying precision tasks and perhaps underlying neural work processes and immediate memories.
\end{abstract}

\section{Keywords}

Mathematical-Computational Modelling, Neurophysiological Circuit, Reverberating Circuit, Parallel Circuit

\section{Introduction}

Mathematical modelling has been increasingly present in the fields of Biology 
and Medicine, in the various types of studies [1] [2], and among many examples, we can cite the study of dynamics for the initiation of immune responses, dynamics of the viral transmission between human groups and the evaluation of the efficacy of therapies addressed to cancer [3] [4] [5]. When it comes to the nervous system, mathematical and computational modelling enables data integration from observations and experiments into a theoretical framework, facilitating the exploitation of the functional role of nervous structures, and neuronal circuits [6] [7] [8] [9].

The use of mathematical and computational modelling to analyze circuits and neuronal networks offers a new way to quantitatively characterize morphofunctional patterns [8] [9] [10]-[16].

Currently, detailed maps of connections within human and other animal brains are being generated with the new technologies available, and graph metrics have been used to understand the general organizational characteristics of these structures. Clusters with short distance integrating characteristics formed by groups of circuits have been found in neuronal networks [17] [18] [19] [20]. Much knowledge has been obtained by modelling neurophysiological circuits using experimental data in the simulations to observe the behavior and limitations of the models [9] [11] [14] [15] [16].

The aim of the present study is to contribute to the knowledge about the functioning of the neuronal circuits. Here we present the results from the implementation of a mathematical-computational model based on a complex neurophysiological model consisting of a reverberating neuronal circuit coupled to a parallel neuronal circuit, using graph theory. The signal processing was investigated in each one of the circuits, as well as the mutual influence of the parallel-reverberant coupling. For this, a program was developed in $\mathrm{C}$ language and neurophysiological data obtained in the literature were applied in the computational simulation.

Graph theory is a computational structure used for analyzing network data, and this can be applied to neuronal circuits at different spatial levels [19] [21] [22]. According to graph theory, structural brain networks can be described as graphs that are composed of nodes (vertices) denoting neural elements (neurons or brain regions), which are connected by edges representing physical connections (axonal projections at synapses) [23].

Reverberant and parallel circuits are built so that the output neuron fires repeatedly for each input signal. The reverberating circuit present feedback loop(s) and form the basis for several rhythmic activities of the central nervous system, as it is capable of making a single input signal reverberate for seconds, minutes or even hours [24] [25].

In a parallel circuit, neurons are organized forming a series and each of these neurons supplies branch to a single final neuron. The parallel circuit seems suitable for the control of neural activities related to precision tasks, such as mathematical calculations, while the reverberating circuit would be related to the maintenance of rhythmic activities, such as breathing movement and natural 
walking [25]. Studies using a decerebrate cat suggested the involvement of reverberating neuronal activity in the conditioning of the soleus muscle [26], such kind of circuit has been found in other central nervous system areas, including the cerebellum-pontine connections [27], and corticothalamic pathways [28].

Ress et al. (2016) [20] identified in the rodent hippocampal formation a highly specialized neuronal topology able to minimize communication cost, which included the prominence of a reverberating circuit with trisynaptic loop. According to Wang [29], the stimulus-specific persistent neural activity would be the neural process subjacent to active (working) memory, i.e., the mnemonic activity would result from synaptic reverberation in a recurrent circuit. More recently, this hypothesis has been tested by some authors [30] [31].

Like reverberating circuits, parallel circuits have been found in brain areas. Hosoya (2019) [32] identified neurons in certain cortical layer organizes into a structure of functional microcolumns, suggesting that parallel processing can be associated with cortical functions, such as sensory perception, motor control, and language processing. Giardino et al. [33] found parallel circuits in the brain connecting the bed nuclei of stria terminalis to the lateral hypothalamus, and they suggested that the circuits would drive opposing emotional states.

\section{Adopted Model}

A neuron can be seen as a digital processor, since it modulates the various information that it receives in the form of action potentials, before conducting any data to other neurons, inside or outside the circuit where it is inserted. Understanding the mechanisms involved in the associative, motor and sensory functions of the brain basically depends on understanding the biodynamics of cortical and subcortical circuits.

It is known that the time of repetitive discharge time in parallel and reverberating circuits depends on the number of neurons compounding these circuits, since each synapse represents a delay time in the information path. Depending on the characteristics of the neurons and their connections (trigger threshold, refractory period and synaptic delay), the circuit can amplify or reduce the input signal power, maintaining the information, or it can change the input signal, modifying completely the information.

The neurophysiological model of a complex neuronal circuit of repetitive discharges built for our study is shown in Figure 1. This consists of two coupled neuronal circuits: 1) a reverberating circuit and 2) a parallel circuit. We can see in this figure that the nervous impulse entering through $E$ can simultaneously propagate through circuit 1 and circuit 2 .

In circuit 1 , reverberating circuit, a signal entering via $A_{1}$ is processed by the neuron $N 1$, and the $N 1$-output passes to $N 2$. Then, $N 2$-output signals leave the circuit by $S 1$ and also return to $N 1$ through the $A_{2-1}$. The synapses between these two neurons are also excitatory. Thus, a reverberating process is formed. For each signal reaching neuron $N 2$, one signal can return to neuron $N 1$, which after 


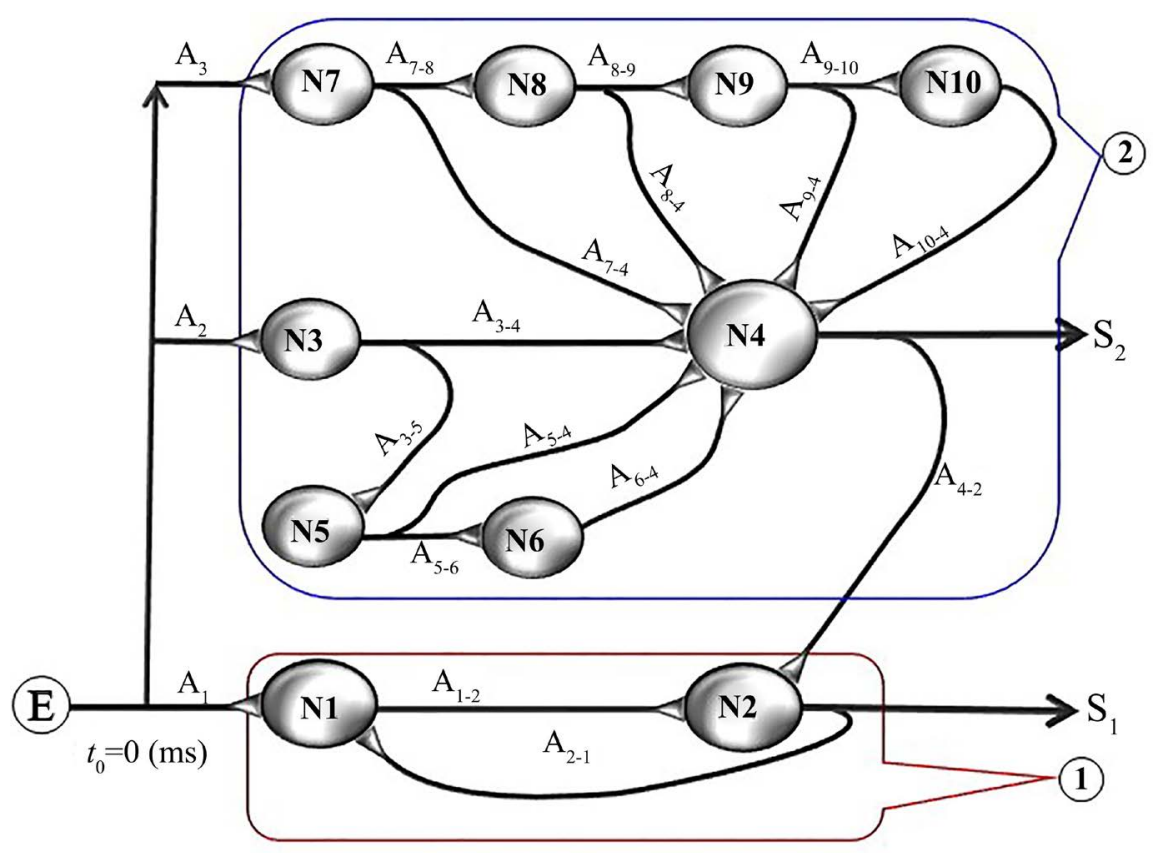

Figure 1. Adopted model for repetitive discharge neuronal circuit.

being processed, $N 1$-output pass to neuron $N 2$, and so on, making the circuit reverberates for some time.

Circuit 2 is a parallel circuit and generates repetitive discharges because signals entering through $A_{2}$ and $A_{3}$ pass from one neuron to another neuron, being processed in each one, and each neuron supplies signals to the neuron N4. All synapses in the circuit are excitatory, and output signs generated in neuron $N 4$, by the processing of inputs from neurons $N 3, N 4, N 5, \ldots, N 10$, leave the circuit through $S 2$.

The axon $A_{4-2}$ connects circuit 2 to circuit 1 , and neuron $N 2$ receives signals coming from neuron $N 4$, being the synapse between them inhibitory. Thus, neuron $N 2$ processes excitatory inputs coming from neuron $N 1$ and inhibitory inputs from neuron $N 4$. The signals from the processing in neuron $N 2$ leaves the circuit by $S 2$ and also return to neuron $N 1$. $S 1$-outputs result from the coupled processing of reverberant and parallel circuits.

In Figure 2, we can see the adopted neurophysiological model (Figure 1) under the graph form. The edges represent the axons and the nodes being the points of contact at the neuronal bodies. Synaptic transmission occurs through the nodes. Each node connects at least two edges, input and output edges. The edge between circuits 2 and 1 (corresponding to axon $A_{4-2}$ ) has a switch to open and close the connection between the two circuits. We considered the circuit occupying a cortical area equivalent to $10 \mathrm{~mm}^{2}$.

For the graph model was considered that: (a) the train of nervous impulse was represented as $(1,0)$-matrix, being $1=$ signal, $0=$ no signal; (b) rhythm and frequency of signal propagation could vary, depending on the distribution of 0 and 1 in matrix; (c) duration of each nervous impulse was $1 \mathrm{~ms}$; (d) in each node, 


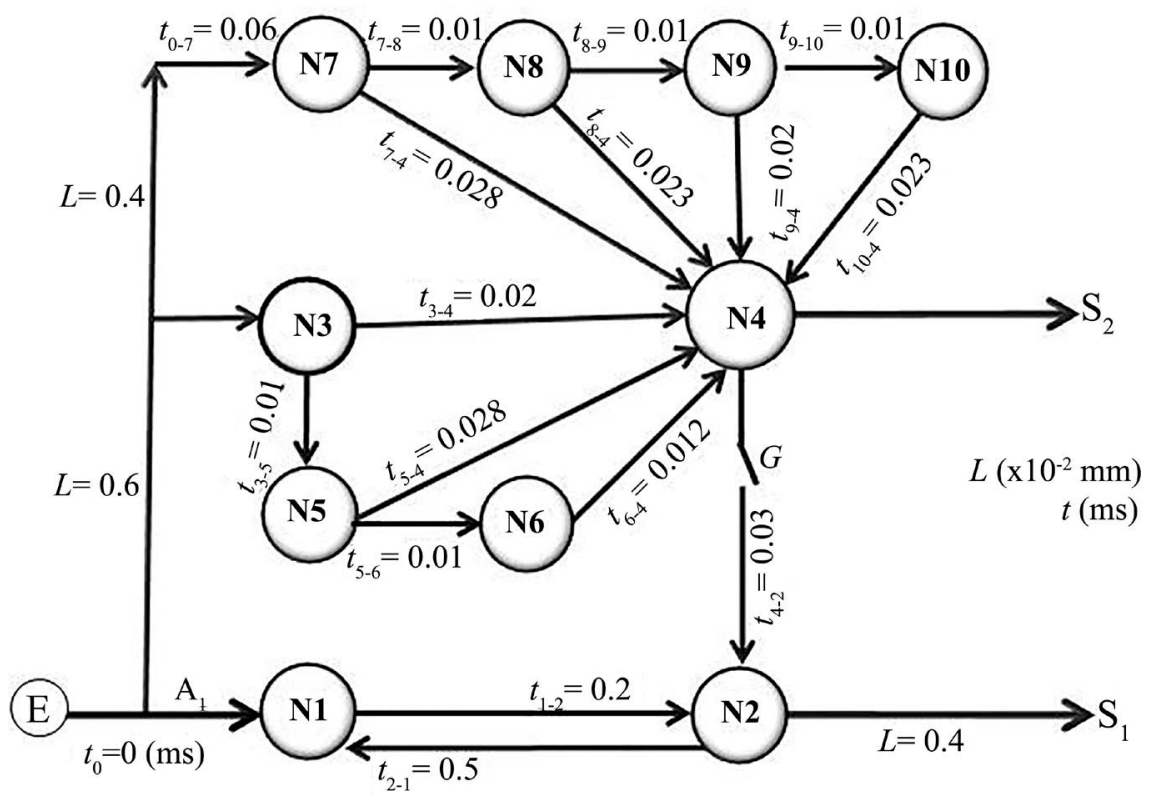

Figure 2. Adopted physiological model (Figure 1) in the Graph form, showing the propagation time of the nerve signals, $t_{i-p}$ in each part of the circuit, where $i$ refers the origin node and $j$ represents destination node. $L=$ distances traveled by the signal from a node to other node.

between each input signal and subsequent output signal there was an interval of $2 \mathrm{~ms}$, corresponding the synaptic delay; (e) time of signal propagation from a node to other depended on the distance between nodes and propagation velocity in edge (axon); (f) propagation speed adopted was $20 \mathrm{~m} / \mathrm{s}$; (g) edge-to-node contacts could be positive or negative; the positive contact representing the excitatory synapse, and negative contact representing inhibitory synapse; (h) at each node, the simultaneous inputs were algebraically added, until the summation reached the threshold value (20 positive pulses), when a nervous impulse would then be triggered by the neuron (or node), and output signal followed by its exit edge.

It was also considered that an input signal could be computed in algebraic summation only if the time interval between its arrival and the last sum did not exceed $40 \mathrm{~ms}$. When the time interval between two signals exceeded this value and the algebraic sum of the signals had not reached the triggering threshold, the sum would start again, being then neglected all those had been added up.

\section{Results}

Implementing our model in $\mathrm{C}++$ and applying neurophysiological values found in the literature, we seek to study the discharge pattern of the reverberant circuit and the parallel circuit separately for the same input signal pattern, examining the influence of the refractory period and the synaptic delay on the respective patterns about to leave. Then, the same study was performed for the complete circuit, that is, in which the two circuits were coupled, and the parallel circuit 
could then influence the functioning of the reverberant.

Figure 3 shows the spectra corresponding to $S 1$-output, being open switch $G$, for 5 pulses in a total of 100 pulses ( 1 pulse every $5 \mathrm{~ms}$ ) entering by $E$, considering two different values of refractory period $(R P)$ : (a) $5 \mathrm{~ms}$ and (b) $2 \mathrm{~ms}$. The synaptic delay $(S D)$ used was $2 \mathrm{~ms}$ in all nodes. Output frequencies were $97 \mathrm{~Hz}$, for $R P=5 \mathrm{~ms}$, and $354 \mathrm{~Hz}$, for $R P=2 \mathrm{~ms}$, and the first pulse arose at $212 \mathrm{~ms}$ and $77 \mathrm{~ms}$ after the first input signal for $R P$ values of $5 \mathrm{~ms}$ and $2 \mathrm{~ms}$, respectively.

Figure 4 presents spectra of $S 2$-output, being open switch $G$, for the same E-input frequency, $R P$ and $S D$ values used in Figure 3. The output frequencies were $105 \mathrm{~Hz}$, for $R P=5 \mathrm{~ms}$ (Figure $4(\mathrm{a})$ ), and $338 \mathrm{~Hz}$, for $R P=2 \mathrm{~ms}$ (Figure $4(\mathrm{~b}))$, and the first pulse arose at $152 \mathrm{~ms}$ and $78 \mathrm{~ms}$ after the first input signal for $R P$ values of $5 \mathrm{~ms}$ and $2 \mathrm{~ms}$, respectively.

Figure 5 illustrates the $S 1$-output spectrum corresponding to the signal processing of the complex neuronal circuit, i.e., when the switch $G$ was closed, for same $E$-input frequency, $R P$ and $S D$ values. Thus, in this figure, the output of

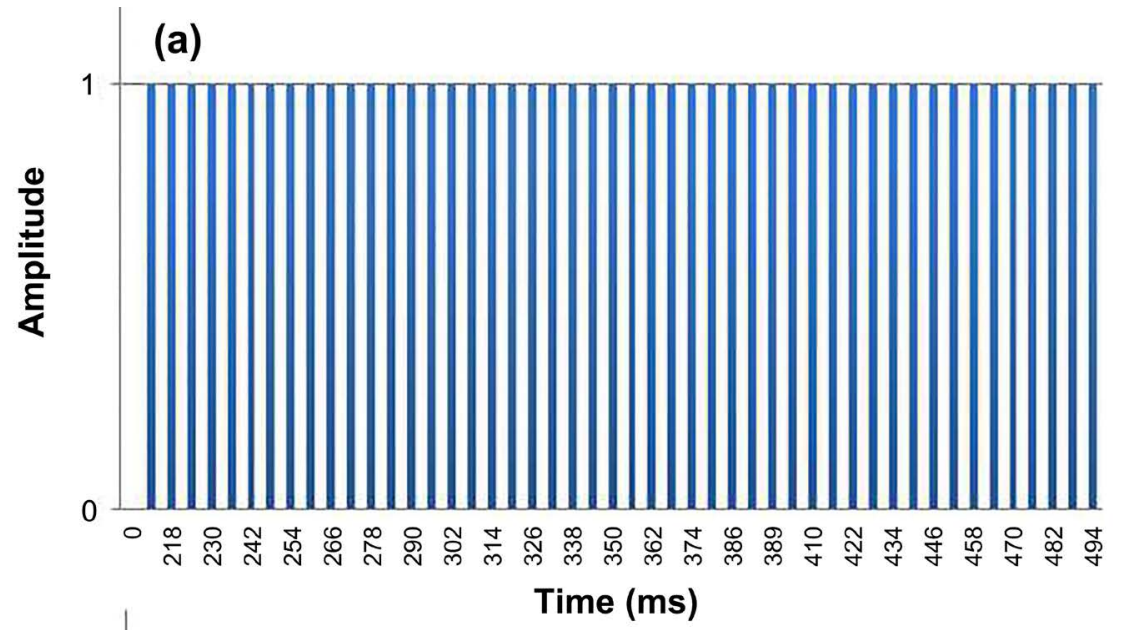

(b)

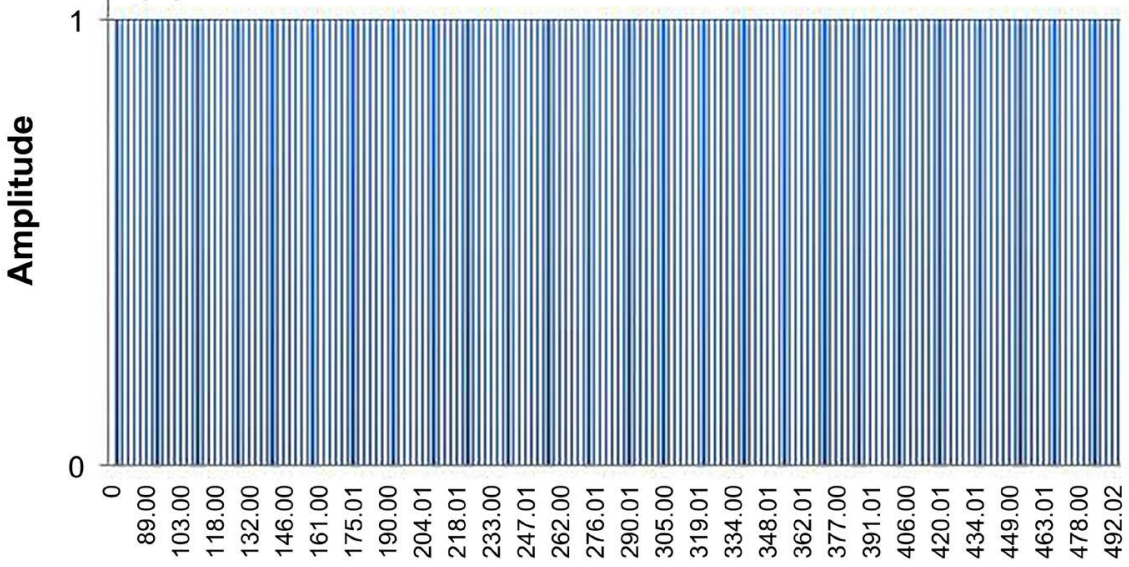

Time (ms)

Figure 3. Spectra corresponding to $S 1$-outputs, being open switch $G$, in response of 5 pulses in a total of 100 pulses (1 pulse every $5 \mathrm{~ms}$ ) entering by $E$, for (a) $5 \mathrm{~ms}$ and (b) 2 ms. $S D=2 \mathrm{~ms}$. 

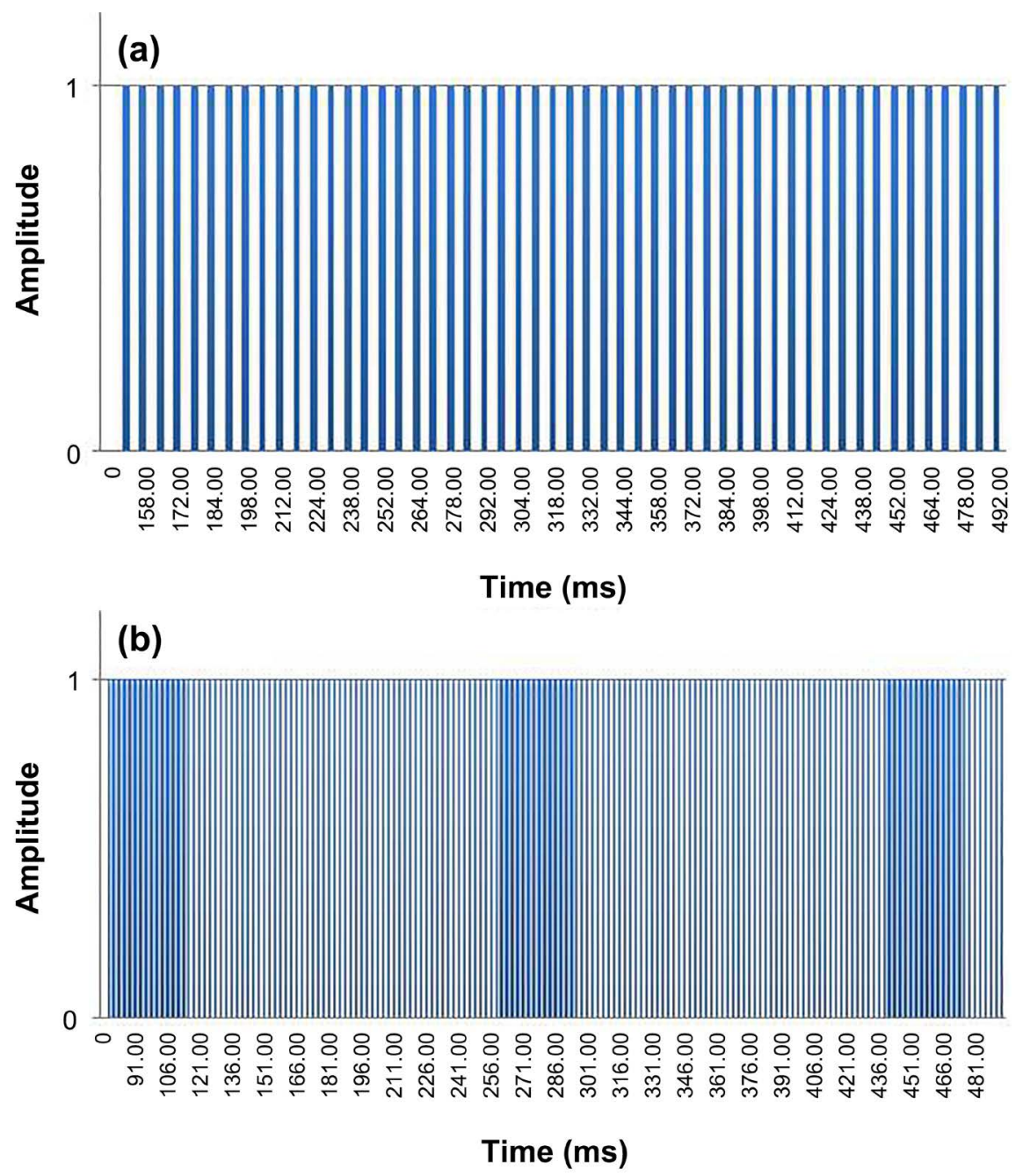

Figure 4. Spectra corresponding to $S 2$-outputs, being open switch $G$, in response of 5 pulses in a total of 100 pulses ( 1 pulse every $5 \mathrm{~ms}$ ) entering by $E$, for (a) $5 \mathrm{~ms}$ and (b) 2 ms. $S D=2 \mathrm{~ms}$.

reverberating circuit was under the direct influence of the processing parallel circuit. For $R P=5 \mathrm{~ms}$ (Figure 5(a)), the output frequency at $S 1$ was $57 \mathrm{~Hz}$ and the first peak arose at $216 \mathrm{~ms}$ after the first E-input. For $R P=2 \mathrm{~ms}$ (Figure 5(b)), the output frequency was $328 \mathrm{~Hz}$ and the first peak arose $106 \mathrm{~ms}$ after the first input pulse.

\section{Discussion}

The program developed using graph theory to represent the adopted physiological model was able to simulate the behavior of a complex neuronal circuit for the specified conditions. The model was tested for two different $R P$ values, 5 and $2 \mathrm{~ms}$, and $S D=2 \mathrm{~ms}$, which are in the scale of values found in experimental studies, as shown by the literature. Refractory period is the period of time after triggering a nervous impulse, during which the neuron is incapable to generate another impulse [25] [34]. 


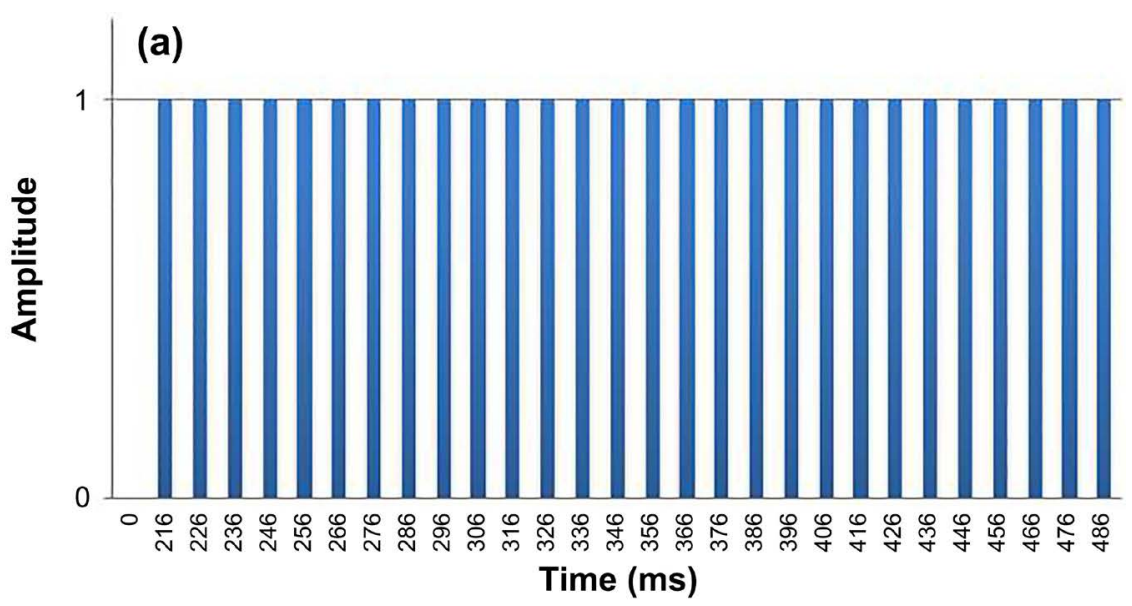

(b)

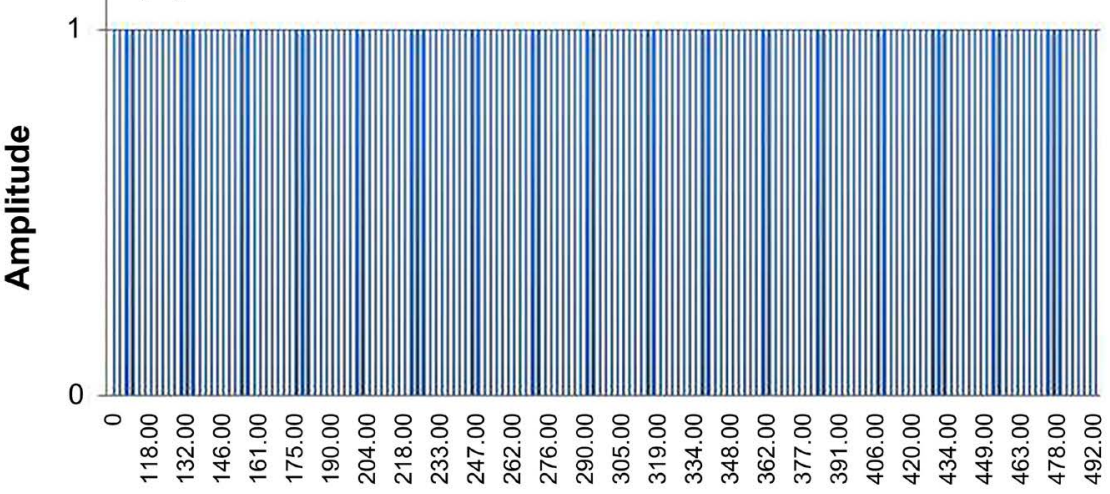

Time (ms)

Figure 5. The spectrum generated at $S 1$-output of the complex neuronal circuit, i.e., two circuits connected via $A_{4-2}$, for 5 input peaks, for two $R P$-values: $5 \mathrm{~ms}$ (a) and $2 \mathrm{~ms}$ (b). $S D$ $=2 \mathrm{~ms}$.

For a stimulation of one pulse every $5 \mathrm{~ms}$, important change was observed in the reverberating activity free of the influence of the parallel circuit with the reduction in $R P$ (Figure 3). For $R P=5 \mathrm{~ms}$ (Figure 3(b)), the $S 1$-output frequency (97 Hz) was about 3.6 times lower $(354 \mathrm{~Hz}$ ) than the value observed for $P R=2$ ms (Figure 3(a)). It means that a 2.5 -fold increase in the $R P$ value caused a reduction of more than $260 \%$ in the output frequency. The change observed in the $S 1$-output frequency was due, in part, to the change in the time required for arising the first pulse, which initiated the reverberating process. Increasing $R P$, the time required to trigger the first pulse decreased 2.7 times.

These results of computational modelling using the graph theory were different from those observed in a previous study [9]. In that study, we implement a mathematical model of synaptic transmission connecting neurons in a circuit of reverberating discharges, which was treat as a neural network. The postsynaptic dynamics was represented by an exponential equation, and for a single input signal, the circuit reverberated at least 1 second. Despite the similarity between the two circuits and of magnitude order of used values in simulations, such 
phenomenon was not observed in the present modelling.

The comparison of spectra in Figure 3 with those in Figure 5 shows the influence of the inhibitory effect of circuit 2 on circuit 1 . For the same E-input (1 pulse every $5 \mathrm{~ms}$ ), the $S 1$-output frequency decreased for both $R P$ values. For $R P$ $=5 \mathrm{~ms}$, Figure 5(a), the $S 1$-output frequency fell from $97 \mathrm{~Hz}$ to $57 \mathrm{~Hz}$, which means a reduction of about $41 \%$. For $R P=2 \mathrm{~ms}$, Figure $5(\mathrm{~b})$, the output frequency reduction was $7 \%$. As for the first output pulse, for $R P=5$, this occurred $2 \%$ faster than observed in Figure $3(\mathrm{a})$, and for $R P=2 \mathrm{~ms}$, it was $27 \%$ faster than observed in Figure 3 (b). The 2.5-fold reduction in the $R P$ value in the complex circuit model caused an increase of 5.8 in the $S 1$-output frequency and the first output pulse was 2 -fold faster.

The reverberating periods observed in the present study were in the order of magnitude of those found by in reverberating activity in lateral amygdala (a brain basal nucleus) in awake rats, which may reach $240 \mathrm{~ms}$ [35].

In the previous study mentioned above, we tested the ability of a reverberating neuronal circuit, similar that included in the complex circuit shown in Figure 1, to keep the triggering rate fixed, i.e., we tested the capacity to preserve the input information. That model followed the dynamics of neural networks, in which the synaptic weight is an important parameter. In the biological view, the synaptic weight represents the strength of each synapse within the set of synapses acting on a neuron [36]. In that mathematical model, the prolonged reverberation of at least 1 second was controlled by varying the synaptic weight over time at the feedback loop synapse. Thus, when the input frequency in the loop synapse reached a certain value, the synaptic weight began to decrease exponentially, adjusting the output rate of the circuit and preserving the information. Thus, we cited some examples of biological synaptic mechanisms that could be being represented by what we called synaptic weight.

In the current model, we try to reproduce the synaptic characteristics (refractory period, synaptic delay, threshold response, spatial and temporal summation), but within the graphs modelling, neglecting the typical analog dynamics of the variation in the amplitudes of postsynaptic potential in the synapses (node).

Adopted conditions obtained from the literature (nervous impulse duration of $1 \mathrm{~ms}$; synaptic delay of $2 \mathrm{~ms}$, propagation speed of $20 \mathrm{~m} / \mathrm{s}, 20$ pulses for trigger threshold, etc.) were appropriate. It is important to note that the inhibitory parallel-reverberant coupling was efficient in controlling the reverberation. The parallel circuit activity was able to reduce the discharge rate of the reverberating circuit from $41 \%$ to $R P=5 \mathrm{~ms}$.

The model brings a new view of the parallel circuit, as a mechanism capable of controlling the reverberation. There is a set of variable parameters in the parallel circuit (in special the number of neurons in series) that can be adjusted, making the circuit able to function as a controller of reverberating circuits in its various functions in the central nervous system, including rhythmic motor activities [25].

In addition to the function that has been attributed in literature, the parallel 
circuit can also be involved in the control of the reverberation for motor activities subjacent the precision tasks, as well as neural process subjacent to working and immediate memories, which would result from synaptic reverberation in a recurrent circuit [29] [30] [31] [35] [37] [38].

\section{Conclusions}

Our computational model using graph theory proved to be able to simulate the dynamics of a complex neuronal circuit composed of a parallel circuit coupled to the reverberant circuit.

The results showed that the refractory period played an important role in forming the pattern of the output spectrum of a reverberating circuit. For uncoupled reverberating and parallel circuits, an increase of 2.5 times in value of this parameter caused a reduction of more than $260 \%$ in the reverberation frequency ( $S 1$-output) and $60 \%$ the parallel circuit output frequency ( $S 2$-output), in addition, there was a delay longer than $100 \mathrm{~ms}$ of the first output signal in both circuits.

As for the complex circuit output, being both circuits, reverberating and parallel, coupled and powered by the same input signal, we observed that the inhibitory action of the parallel circuit was able to regulate the reverberation frequency, suggesting that parallel circuits may be controlling reverberation circuits related to motor activities subjacent the precision tasks, and maybe in neural process subjacent to working and immediate memories.

\section{Acknowledgements}

Authors are grateful to CNPq (National Council for Scientific and Technological Development) for the financial support with scholarship.

\section{Conflicts of Interest}

The authors declare no conflicts of interest regarding the publication of this paper.

\section{References}

[1] Röhrle, O., Yavuz, U.Ş., Klotz,T., Negro, F. and Heidlauf, T. (2019) Multiscale Modeling of the Neuromuscular System: Coupling Neurophysiology and Skeletal Muscle Mechanics. WIREs Systems Biology and Medicine, 11, e1457.

https://doi.org/10.1002/wsbm.1457

[2] Cutsuridi, V. (2019) Memory Prosthesis: Is It Time for a Deep Neuromimetic Computing Approach? Frontier in Neuroscience, 13, Article No. 667. https://doi.org/10.3389/fnins.2019.00667

[3] Li, X., Zhong, C.Q., Yin, Z., Qi, H., Xu, F., He, Q. and Shuai, J. (2020) Data-Driven Modeling Identifies TIRAP-Independent MYD88 Activation Complex and Myddosome Assembly Strategy in LPS/TLR4 Signaling. International Journal of Molecular Sciences, 21, 3061. https://doi.org/10.3390/ijms21093061

[4] Khajji, B., Kada, D., Balatif, O. and Rachik, M. (2020) A Multi-Region Discrete 
Time Mathematical Modeling of the Dynamics of Covid-19 Virus Propagation Using Optimal Control. Journal of Applied Mathematics and Computer, 64, 255-281. https://doi.org/10.1007/s12190-020-01354-3

[5] Goris, N.A.V., Rodríguez, J.L.G., González, M.M., Borges, B.O., Morales, D.F., Calzado, E.M., Castañeda, A.R.S., Torres, L.M., Montijano, J.I., González, V.G.S., Pérez, D.J., Posada, O.O., Martínez, J.A., Delgado, A.G., Martínez, K.G., Mon, M.L., Monzón, K.L., Ciria, H.M.C. and Cabrales, L.E.B. (2020) Efficacy of Direct Current Generated by Multiple-Electrode Arrays on F3II Mammary Carcinoma: Experiment and Mathematical Modeling. Journal of Translational Medicine, 18, Article No. 190. https://doi.org/10.1186/s12967-020-02352-6

[6] Kim, T., Capps, R.A., Hamade, K.C., Barnett, W.H., Todorov, D.I., Latash, E.M., Markin, S.N., Rybak, I.A. and Molkov, Y.I. (2019) The Functional Role of Striatal Cholinergic Interneurons in Reinforcement Learning from Computational Perspective. Frontier of Neural Circuits, 13, Article No. 10.

https://doi.org/10.3389/fncir.2019.00010

[7] Jarvis, S., Nikolic, K. and Schultz, S.R. (2018) Neuronal Gain Modulability Is Determined by Dendritic Morphology: A Computational Optogenetic Study. PLoS Computational Biology, 14, e1006027. https://doi.org/10.1371/journal.pcbi.1006027

[8] Rodrigues, V.F., Kalil, C., de Castro, M.C.S., Silva, D. and Cortez, C.M. (2018) Mathematical-Computational Modeling in Behavior's Study of Repetitive Discharge Neuronal Circuits. In: Alves Barbosa da Silva, F., Carels, N. and Paes Silva Jr., F., Eds., Theoretical and Applied Aspects of Systems Biology, Springer International Publishing AG, Cham, 243-259. https://doi.org/10.1007/978-3-319-74974-7_13

[9] Rodrigues, V.F., Wedemann, R.S., de Castro, M.C.S., Silva, D. and Cortez, C.M. (2020) Behavior of a Model for Feedback-Controlled Reverberating Circuit and Immediate Memory Function. Trends in Computational and Applied Mathenamtics, 21, 601-616.

[10] Huang, C., Ruff, D.A., Pyle, R., Rosenbaum, R., Cohen, M.R. and Doiron, B. (2019) Circuit Models of Low-Dimensional Shared Variability in Cortical Networks. Neuron, 101, 337-348.E4. https://doi.org/10.1016/j.neuron.2018.11.034

[11] Dalcin, B.L.G., Cruz, F.A.O., Cortez, C.M. and Passos, E.L. (2015) Applying Backpropagation Neural Network in the Control of Medullary Reflex Pattern. AIP Conference Proceedings, 1702, Article No. 130006. https://doi.org/10.1063/1.4938913

[12] Dalcin, B.L.G., Cruz, F.A.O., Cortez, C.M. and Passos, E.L. (2005) Computer Modeling of a Spinal Reflex Circuit. Brazilian Journal of Physics, 35, 987-994. https://doi.org/10.1590/S0103-97332005000600013

[13] Sorger, J., Katja Bühler K.B., Schulze, F., Liu, T. and Dickson, B. (2013) NeuroMAP -Interactive Graph-Visualization of the Fruit Fly's Neural Circuit. 2013 IEEE Symposium on Biological Data Visualization, Atlanta, GA, USA, 13-14 October 2013, 73-80. https://ieeexplore.ieee.org/document/6664349/

[14] Cardoso, F.R.G., Cruz, F.A.O., Silva, D. and Cortez, C.M. (2009) Computational Modeling of Synchronization Process of the Circadian Timing System of Mammals. Biological Cybernetics, 100, 385-393. https://doi.org/10.1007/s00422-009-0309-6

[15] Cruz, F.A.O., Silva, D. and Cortez, C.M. (2008) Simulation of Spinal Reflex Circuit Model Controlled by a Central Pattern Generator. Far East Journal of Applied Mathematics, 33, 307-336.

[16] Cruz, F.A.O. and Cortez, C.M. (2005) Computer Modeling of a Spinal Reflex Circuit. Physica A: Statistical Mechanics and its Applications, 353, 258-270.

https://doi.org/10.1016/j.physa.2004.12.046 
[17] Arnatkevičiūte, A., Fulcher, B.D. and Fornito, A. (2019) Uncovering the Transcriptional Correlates of Hub Connectivity in Neural Networks. Frontier of Neural Circuits, 13, Article No. 47. https://doi.org/10.3389/fncir.2019.00047

[18] Li, X.J. and Yang, G.H. (2017) Graph Theory-Based Pinning Synchronization of Stochastic Complex Dynamical Networks. IEEE Transactional on Neural Network and Learning Systems, 28, 427-437. https://doi.org/10.1109/TNNLS.2016.2515080

[19] Meyer-Bäse, A., Roberts, R.G., Illan, I.A., Meyer-Bäse, U., Lobbes, M., Stadlbauer, A. and Pinker-Domenig, K. (2017) Dynamical Graph Theory Networks Methods for the Analysis of Sparse Functional Connectivity Networks and for Determining Pinning Observability in Brain Networks. Frontier in Computational Neurosciences, 11, Article No. 87. https://doi.org/10.3389/fncom.2017.00087

[20] Rees, C.L., Wheeler, D.W., Hamilton, D.J., White, C.M., Komendantov, A.O. and Ascoli, G.A. (2016) Graph Theoretic and Motif Analyses of the Hippocampal Neuron Type Potential Connectome. eNeuro, 3, ENEURO.0205-16. https://doi.org/10.1523/ENEURO.0205-16.2016

[21] Lee, U., Oh, G., Kim, S., Noh, G., Choi, B. and Mashour, G.A. (2010) Brain Networks Maintain a Scale-Free Organization across Consciousness, Anesthesia, and Recovery: Evidence for Adaptive Reconfiguration. Anesthesiology, 113, 1081-1091. https://doi.org/10.1097/ALN.0b013e3181f229b5

[22] Stobb, M., Peterson, J.M., Mazzag, B. and Gahtan, E. (2012) Graph Theoretical Model of a Sensorimotor Connectome in Zebrafish. PLoS ONE, 7, e37292. https://doi.org/10.1371/journal.pone.0037292

[23] Bullmore, E. and Sporns, O. (2009) Complex Brain Networks: Graph Theoretical Analysis of Structural and Functional Systems. Nature Reviews Neuroscience, 186, 187-198. https://doi.org/10.1038/nrn2575

[24] Wilting, J. and Priesemann, V. (2019) Between Perfectly Critical and Fully Irregular: A Reverberating Model Captures and Predicts Cortical Spike Propagation. Cerebral Cortex, 29, 2759-2770. https://orcid.org/0000-0001-6880-5401

[25] Carpenter, R. and Reddi, B. (2012) Neurophysiology. A Conceptual Approach. CRC Press, London. https://doi.org/10.1201/b13510

[26] Hultborn, H., Wigström, H. and Wängberg, B. (1975) Prolonged Activation of Soleus Motoneurones Following a Conditioning Train in Soleus Ia Afferents-A Case for a Reverberating Loop? Neuroscience Letters, 1, 147-152. https://doi.org/10.1016/0304-3940(75)90030-0

[27] Tsukahara, N. (1972) The Properties of the Cerebello-Pontine Reverberating Circuit. Brain Research, 40, 67-71. https://doi.org/10.1016/0006-8993(72)90108-4

[28] Hsiang-Tung, C. (1950) The Repetitive Discharges of Corticothalamic Reverberating Circuit. Journal of Neurophysiology, 13, 235-257.

https://doi.org/10.1152/jn.1950.13.3.235

[29] Wang, X.J. (2001) Synaptic Reverberation Underlying Mnemonic Persistent Activity. Trends in Neuroscience, 24, 455-463. https://doi.org/10.1016/S0166-2236(00)01868-3

[30] Bliss, D.P. and D’Esposito, M. (2017) Synaptic Augmentation in a Cortical Circuit Model Reproduces Serial Dependence in Visual Working Memory. PLoS ONE, 12, e0188927. https://doi.org/10.1371/journal.pone.0188927

[31] Watanabe, Y. and Funahashi, S. (2012) Thalamic Mediodorsal Nucleus and Working Memory. Neuroscience \& Biobehavioral Reviews, 36, 134-142.

https://doi.org/10.1016/j.neubiorev.2011.05.003 
[32] Hosoya, T. (2019) The Basic Repeating Modules of the Cerebral Cortical Circuit. Proceedings of the Japan Academy. Series B, Physical and Biological Sciences, 95, 303-311.

[33] Giardino, W.J., Eban-Rothschild, A., Christoffel, D.J., Li, S.B., Malenka, R.C. and de Lecea, L. (2018) Parallel Circuits from the Bed Nuclei of Stria Terminalis to the Lateral Hypothalamus Drive Opposing Emotional States. Nature Neuroscience, 21, 1084-1095. https://doi.org/10.1038/s41593-018-0198-x

[34] Guyton, A.C. and Hall, J.E. (2006) Textbook of Medical Physiology. 11th Edition, Elsevier Saunders, Philadelphia.

[35] Johnson, L.R., Ledoux, J.E. and Doyère, V. (2009) Hebbian Reverberations in Emotional Memory Micro Circuits. Frontier in Neuroscience, 3, 198-205.

https://doi.org/10.3389/neuro.01.027.2009

[36] Friesen, W.O. and Block, G.D. (1984) What Is a Biological Oscillator? American Journal of Physiology, 246, R847-R853. https://doi.org/10.1152/ajpregu.1984.246.6.R847

[37] Takeuchi, T., Duszkiewicz, A.J. and Morris, R.G.M. (2014) The Synaptic Plasticity and Memory Hypothesis: Encoding, Storage and Persistence. Philosophical Transaction of the Royal Society of London B Biological Sciences, 369, Article ID: 20130288. https://doi.org/10.1098/rstb.2013.0288

[38] Pereira, J. and Wang, X.J. (2015) A Tradeoff between Accuracy and Flexibility in a Working Memory Circuit Endowed with Slow Feedback Mechanisms. Cerebral Cortex, 25, 3586-601. https://doi.org/10.1093/cercor/bhu202 